\title{
Blue supergiant progenitors from binary mergers for SN 1987A and other Type II-peculiar supernovae
}

\author{
Athira Menon $^{1}$ and Alexander Heger ${ }^{1,2,3}$ \\ ${ }^{1}$ Monash Centre for Astrophysics (MoCA) and School of Physics and Astronomy, Monash \\ University, Clayton, VIC 3800, Australia \\ email: athira.menon@monash.edu \\ ${ }^{2}$ School of Physics and Astronomy, University of Minnesota, Minneapolis, MN 55455, U.S.A. \\ ${ }^{3}$ Centre for Nuclear Astrophysics, Shanghai Jiao Tong University, Shanghai, China \\ email: alexander.heger@monash.edu
}

\begin{abstract}
We present results of a systematic and detailed stellar evolution study of binary mergers for blue supergiant (BSG) progenitors of Type II supernovae, particularly for SN 1987A. We are able to reproduce nearly all observational aspects of the progenitor of SN 1987A, Sk $-69^{\circ} 202$, such as its position in the HR diagram, the enrichment of helium and nitrogen in the triple-ring nebula and its lifetime before its explosion. We build our evolutionary model based on the merger model of Podsiadlowski et al. (1992), Podsiadlowski et al. (2007) and empirically explore an initial parameter consisting of primary masses, secondary masses and different depths up to which the secondary penetrates the He core during the merger. The evolution of the post-merger star is continued until just before iron-core collapse. Of the 84 pre-supernova models $\left(16 \mathrm{M}_{\odot}-23 \mathrm{M}_{\odot}\right)$ computed, the majority of the pre-supernova models are compact, hot BSGs with effective temperature $>12 \mathrm{kK}$ and $30 \mathrm{R}_{\odot}-70 \mathrm{R}_{\odot}$ of which six match nearly all the observational properties of Sk $-69^{\circ} 202$.
\end{abstract}

Keywords. (stars:) supernovae: individual (SN 1987A), stars: evolution, (stars:) binaries (including multiple): close

\section{Introduction}

The remarkable supernova SN 1987A that exploded in the Large Magellanic Cloud (LMC) (West et al. 1987) was an unusual Type II SNe- it had a dome-shaped light curve (Catchpole et al. 1988, Hamuy et al. 1988), unlike typical Type II-P SNe. The compact BSG progenitor that exploded as SN 1987A, was a B3 Ia supergiant in the LMC, called Sk $-69^{\circ} 202$ (Walborn et al. 1987, Blanco et al. 1987). Another surprising aspect of the progenitor was the presence of a triple-ring nebula of circumstellar material, enriched in helium and nitrogen (Lundqvist \& Fransson 1996, France et al. 2011), ejected by the progenitor about 15-20 kyr before the explosion (Wampler et al. 1990, Burrows et al. 1995, Crotts et al. 2000). The angular momentum required for the expulsion of the nebular material and the formation of its complex geometry, could only have been possible from a binary merger (Podsiadlowski 1992, Morris \& Podsiadlowski 2007, Morris \& Podsiadlowski 2009).

There has not been so far, an evolutionary model that can consistently reproduce all the key signatures of the progenitor of SN 1987A (Smartt 2009), despite the many attempts from single star models. Podsiadlowski et al. 1992, and Podsiadlowski et al. 2007, described a binary merger scenario for the progenitor, that could explain all the observations of $\mathrm{Sk}-69^{\circ} 202$. In these works, they demonstrated that the merger of a $15 \mathrm{M}_{\odot}$ primary red supergiant (RSG) with a main-sequence secondary of $5 \mathrm{M}_{\odot}$ could 

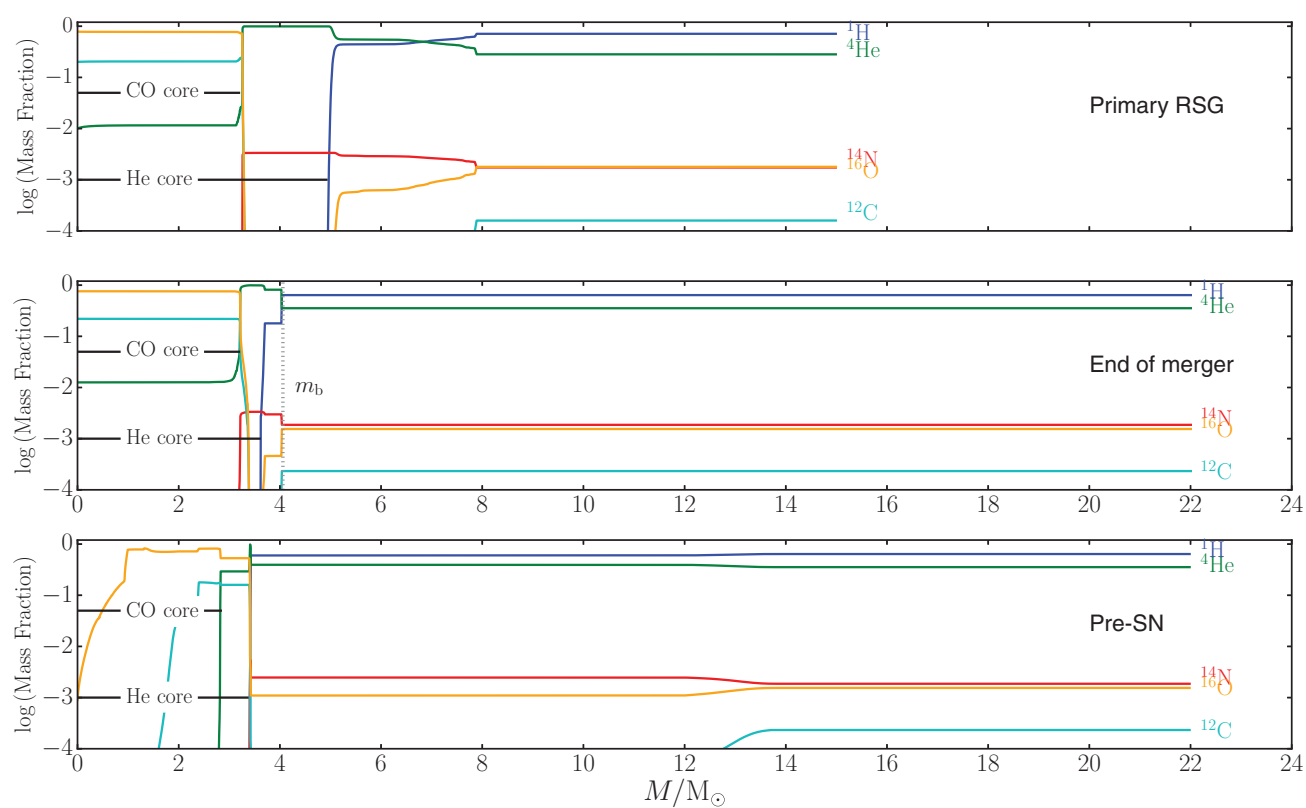

Figure 1. Top panel: composition of the primary RSG model of $M_{1}=16 \mathrm{M}_{\odot}$ just prior to the merger. Middle Panel: composition at the end of the merger with $M_{2}=7 \mathrm{M}_{\odot}$, for mixing boundary $m_{\mathrm{b}}$ (dotted vertical line) set for $f_{\mathrm{sh}}=50 \%$. Bottom panel: Composition of pre-SN model.

lead to a BSG, whose explosion would resemble SN 1987A. The scenario begins with a wide binary system of a $15 \mathrm{M}_{\odot}-20 \mathrm{M}_{\odot}$ primary and a $1 \mathrm{M}_{\odot}-5 \mathrm{M}_{\odot}$ secondary, with an initial orbital period greater than $10 \mathrm{yr}$. When the primary evolves to an RSG with a $\mathrm{CO}$ core, it transfers mass on a dynamically unstable timescale to the secondary mainsequence star. A common envelope (CE) phase is ensued and the envelope is partially ejected. The secondary star is engulfed by the CE and spirals in towards the core of the primary. Eventually the secondary undergoes a Roche Lobe Overflow and is completely dissovled in the envelope. Hydrodynamic simulations of the merger showed the He core being penetrated by the secondary, shrinking it in the process and resulting in the dredgeup of He in the envelope (Ivanova et al. 2002). The merger lasts for an order of $\sim 100 \mathrm{yr}$ and the post-merger star could evolve to a BSG (Ivanova \& Podsiadlowski 2002).

\section{Methodology}

We construct an evolutionary model based on the above merger scenario, over an initial parameter consisting of the main-sequence primary mass, $M_{1}=15,16,17 \mathrm{M}_{\odot}$ with an initial rotation velocity of $v / v_{\text {cri }}=0.30$, main-sequence secondary mass, $M_{2}=2,3, \ldots, 8 \mathrm{M}_{\odot}$ and fraction of the He shell from the He core dredged up, $f_{\mathrm{sh}}=10,50,90,100 \%$. We evolve 84 such initial models until just prior to the core-collapse stage (the pre-supernova (pre-SN) model), using KePLER, an implicit one-dimensional hydrodynamics code that can compute stellar evolution models with rotation and nucleosynthesis (Heger et al. 2000, Heger et al. 2005, Woosley et al. 2002). The initial (solar-scaled) composition is that of the LMC $-X_{\mathrm{H}}=0.739, X_{\mathrm{He}}=0.255$ and $Z=0.0055$ (Brott et al. 2011).

We compare the pre-SN models computed with the three confirmed signatures of $\mathrm{Sk}$ $-69^{\circ} 202$ : 

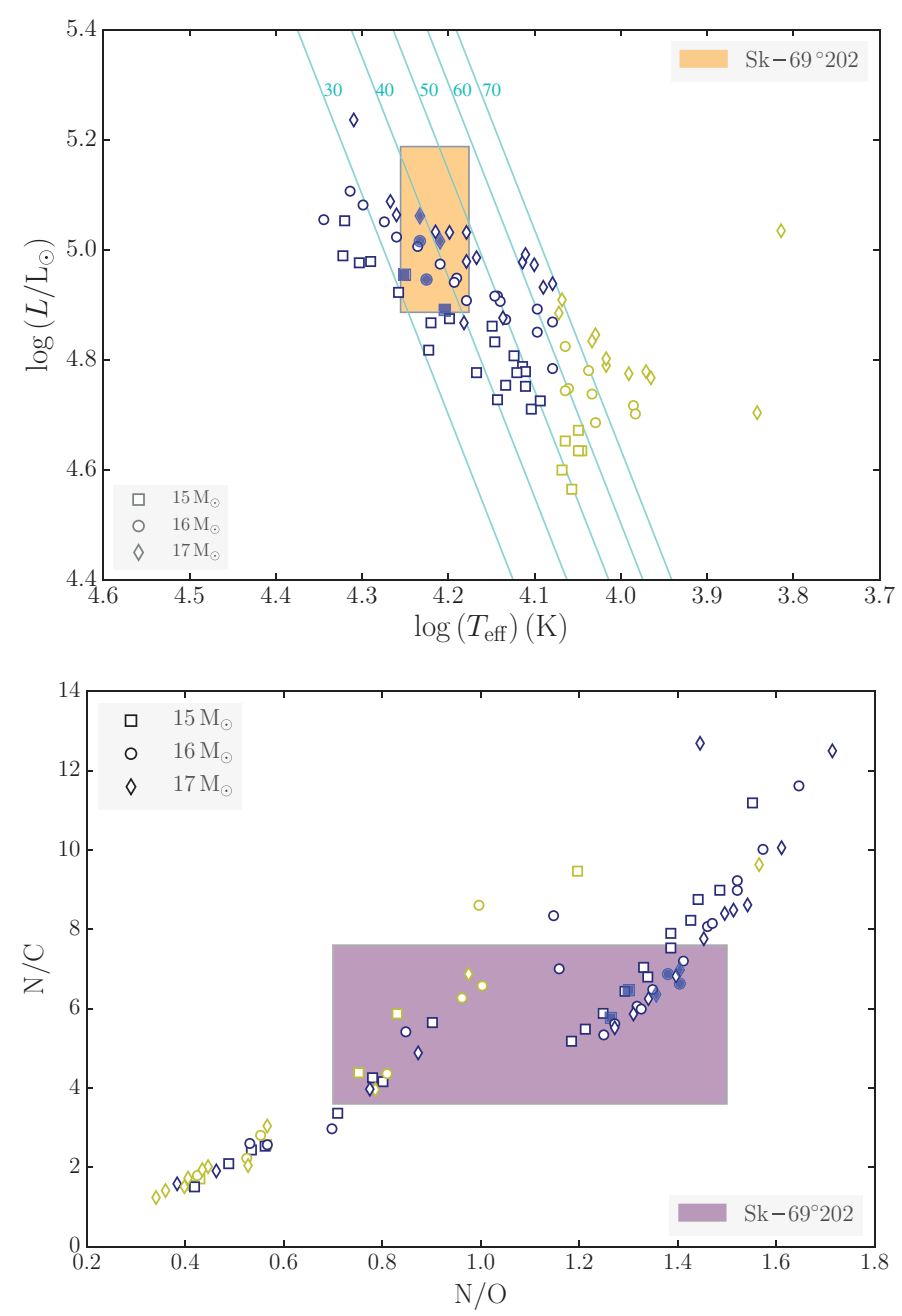

Figure 2. Distribution of all computed pre-SN models in the HR diagram (top) and their relative number ratios of N/C and N/O. Shade regions denote the observational limits for Sk $-69^{\circ} 202$. Yellow symbols are YSGs, blue symbols are BSGs and filled blue symbols are progenitor models for SN 1987A.

(a) Lies in the HR diagram where $\mathrm{Sk}-69^{\circ} 202$ was observed before exploding$\log \left(L / \mathrm{L}_{\odot}\right)=5.15-5.45, T_{\text {eff }}=15 \mathrm{kK}-18 \mathrm{kK}$ and $R / \mathrm{R}_{\odot}=28-58$ (Woosley 1988).

(b) Relative elemental ratios by number in the surface from the BSG model match those of the triple-ring nebula; N/C $\sim 5 \pm 2, \mathrm{~N} / \mathrm{O} \sim 1.1 \pm 0.4$ (Lundqvist \& Fransson 1996) and $\mathrm{He} / \mathrm{H}=0.14 \pm 0.06$ (France et al. 2011).

(c) Lifetime of the post-merger BSG phase before explosion is at least $15 \mathrm{kyr}$.

In this first study, the merger is a simplified process that does not include heating or angular momentum loss from the CE phase. We first choose a primary mass, $M_{1}$ and evolve it to an RSG with a CO core. Since the primary RSG model is rotating, its envelope becomes enhanced in nitrogen and helium. The secondary main-sequence star, $M_{2}$, is evolved to the same age as the primary RSG. $M_{2}$ gets accreted on the primary over $100 \mathrm{yr}$ and is simultaneously mixed throughout its envelope using a numerical prescription. Mixing is restricted to a specified boundary inside the He core, depending on the value set for $f_{\mathrm{sh}}$. The mixing causes the He core to shrink; larger values of $f_{\text {sh }}$ lead to smaller 

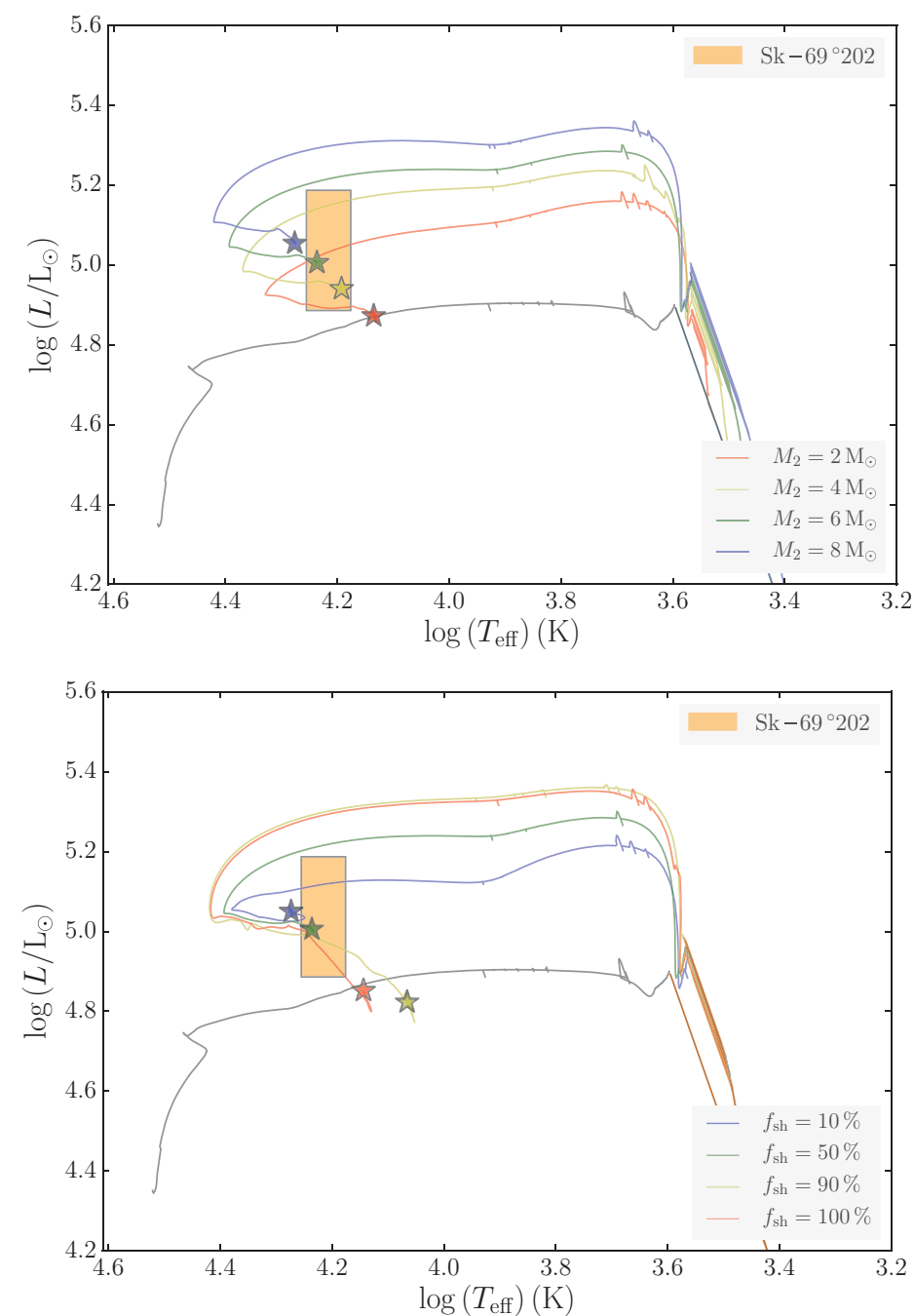

Figure 3. Top: Evolutionary tracks from the merger of $M_{1}=16 \mathrm{M}_{\odot}$ and $M_{2}=6 \mathrm{M}_{\odot}$, with varying values of $f_{\mathrm{sh}}$. Bottom: Tracks from the merger of $M_{1}=16 \mathrm{M}_{\odot}$ and various values of $M_{2}$ keeping $f_{\mathrm{sh}}=50 \%$.

He cores. The envelope is further enriched in He and also mixed with carbon and oxygen dredged up from the He core. At the end of the merger, the post-merger star has a small $\mathrm{He}$ core and a massive envelope with relative enhancements of $\mathrm{N} / \mathrm{C}, \mathrm{N} / \mathrm{O}$ and $\mathrm{He} / \mathrm{H}$ (Fig. 1).

\section{Results}

The majority of pre-SN models amongst the 84 we computed, are BSGs (i.e., have $T_{\text {eff }} \geqslant 12 \mathrm{kK}$ ), while the rest are yellow supergiants (YSGs) $\left(12 \mathrm{kK}<T_{\text {eff }} \leqslant 4 \mathrm{kK}\right)$. Of these, six pre-SN models match the three observational signatures of Sk $-69^{\circ} 202$ (Fig. 2).

The most influential initial parameter that determines how hot the surface of the preSN model is, is $f_{\mathrm{sh}}$. Across the range of $M_{1}$ and $M_{2}$ studied, pre-SN models are blue when the He core does not become smaller than $f_{\mathrm{sh}}=10 \%-50 \%$. These values of $f_{\mathrm{sh}}$ 
also result in values of $\mathrm{N} / \mathrm{C}$ and $\mathrm{N} / \mathrm{O}$ in the surface identical to $\mathrm{Sk}-69^{\circ} 202$. The next parameter of importance is $M_{2}$. Increasing $M_{2}$ for a particular He core mass, increases the $T_{\text {eff }}$ of the pre-SN star (Fig. 3) but decreases N/C and N/O in the surface. Finally, the parameter that affects the lifetime of the BSG star before its explosion, is the age of the primary model at the time of the merger. Younger primary models on the red giant branch lead to longer-lived post-merger BSG models, for a given combination of initial parameters.

We were unable to draw a relation between the three initial parameters, $M_{1}, M_{2}, f_{\mathrm{sh}}$ and the position of the pre-SN model in the HR diagram. The results of Podsiadlowski et al. 1992 indicated that the accretion of $M_{2}$, which led to high envelope to core mass ratios, was sufficient for a star to become blue at the time of explosion. We do not find such a linear relationship between core-envelope mass ratio and $T_{\text {eff }}$ of the pre-SN model (Fig. 3). In fact, if $M_{2}$ is simply accreted in our model without He core dredge-up, the post-merger star remains red until its explosion. There also does not exist any correlation between $\mathrm{N} / \mathrm{C}$ and $\mathrm{N} / \mathrm{O}$ ratios in the surface and the position in the HR diagram of a pre-SN model, implying they are independent constraints (Fig. 2).

The progenitor models for SN 1987A are more massive than their single star counterparts $\left(16 \mathrm{M}_{\odot}-23 \mathrm{M}_{\odot}\right.$ as against $\left.14 \mathrm{M}_{\odot}-20 \mathrm{M}_{\odot}\right)$ and have smaller He cores $\left(2.4 \mathrm{M}_{\odot}-4.5 \mathrm{M}_{\odot}\right.$ as against $4 \mathrm{M}_{\odot}-7 \mathrm{M}_{\odot}$, (Woosley 1988)). In a future study we shall compare the light curves from their explosions to that of SN 1987A.

\section{References}

Brott, I. et al., 2011 AAP, 530

Burrows, C. J. et al. 1995, ApJ, 680, 452

Catchpole, R. M. et al. 1988, MNRAS, 75, 231

Crotts, A. P. S. \& Heathcote, S. R., 2000 ApJ, 426, 528

Hamuy, M., Suntzeff, N. B., Gonzalez, R., \& Martin, G., 1988 AJ, 63, 95

Heger, A., Langer, N., \& Woosley, S. E., 2000 ApJ, 368, 528

Heger, A., Woosley, S. E., \& Spruit, H. C., 2005 ApJ, 350, 626

Ivanova, N., Podsiadlowski, P., \& Spruit, H., 2002 MNRAS, 819, 334

Ivanova, N. \& Podsiadlowski, P., 2002 Astronomical Society of the Pacific Conference Series, 245,279

Morris, T. \& Podsiadlowski, P., 2007 Science, 1103, 315

Morris, T. \& Podsiadlowski, P., 2009 MNRAS, 515, 399

Podsiadlowski, P., Joss, P. C., \& Hsu, J. J. L. 1992, ApJ, 246, 391

Podsiadlowski, P. 1992, PASP, 717, 104

Podsiadlowski, P., Morris, T. S., \& Ivanova, N. 2007, AIPCS, 125, 937

Smartt, S. J., 2009 ARAA, 63, 47

Walborn, N. R., Prevot, M. L., Prevot, L., Wamsteker, W., Gonzalez, R., Gilmozzi, R., \& Fitzpatrick, E. L. 1989, AAP, 229, 219

Woosley, S. E., 1988 ApJ, 218, 330

Woosley, S. E., Heger, A., \& Weaver, T. A., 2002 Reviews of Modern Physics, 1015, 74 